\title{
Simulations of the Local Universe
}

\author{
S. Gottlöber ${ }^{1}$, A. Klypin ${ }^{2}$, A. Kravtsov ${ }^{3}$, Y. Hoffman ${ }^{4}$, and A. \\ Faltenbacher $^{1}$ \\ 1 Astrophysikalisches Institut Potsdam, An der Sternwarte 16, 14482 Potsdam, \\ Germanysgottloeber@aip.de \\ 2 Astronomy Department, New Mexico State University, USA aklypin@nmsu.edu \\ 3 Dept. of Astronomy \& Astrophysics, CfCP, The University of Chicago, USA \\ 4 Racah Institute of Physics, Hebrew University, Jerusalem, Israel
}

\section{Introduction}

One of the greatest challenges of modern astrophysics is understanding how galaxies, such as our Milky Way, form within the framework of the Big Bang cosmology. The current theory of structure formation, the extension of the Big Bang model called the Cold Dark Matter (CDM) scenario, predicts that galaxies form within extended massive dark matter halos built from smaller pieces that collided and merged, resulting in the hierarchy of galaxies, groups, and clusters observed today. The entire sequence of events is thought to be seeded by quantum fluctuations in the very early Universe and governed by mysterious "dark matter" which constitutes about $85 \%$ of all matter in the universe. Although the accurate properties of galaxies depend on complicated baryonic processes (radiative cooling, formation and evolution of stars, etc.) operating on small scales, we expect that overall spatial distribution of dark matter halos is closely related to the observed galaxy distribution. Here we present numerical simulations designed to study the formation, evolution and present day properties of such dark matter halos in different cosmological environments.

In all simulations the spatially flat cold dark matter model with a cosmological constant ( $\Lambda \mathrm{CDM}$ with $\Omega_{\mathrm{M}}=0.3, \Omega_{\Lambda}=0.7, \sigma_{8}=0.9$, and $h=0.7$ ), favored by most current observations, has been assumed.

\section{Numerical simulations}

The Adaptive Refinement Tree (ART) $N$-body code 8, 9] was used to run all numerical simulation analyzed in this paper. In some of the simulations described below the code also included eulerian gasdynamics [10]. The code uses Adaptive Mesh Refinement technique to achieve high resolution in the regions of interests. The computational box is covered with a uniform grid which defines the lowest (zeroth) level of resolution. The code then reaches high force resolution by recursively refining all high density regions using an automated refinement algorithm. This creates an hierarchy of refinement 
meshes of different resolutions, sizes, and geometries covering regions of interest. The refinement data structures and algorithms [4] allow individual cubic cells to be refined. The shape of the refinement mesh can thus effectively match the geometry of the region of interest. This algorithm is well suited for simulations of a selected region within a large computational box, as in the simulations presented below.

During the integration, spatial refinement is accompanied by temporal refinement. Namely, each level of refinement, $l$, is integrated with its own time step $\Delta a_{l}=\Delta a_{0} / 2^{l}$, where $\Delta a_{0}$ is the global time step of the zeroth refinement level. This variable time stepping is very important for accuracy of the results. As the force resolution increases, more steps are needed to integrate the trajectories accurately. In addition to spatial and temporal refinement, simulations described below also use non-adaptive mass refinement to increase the mass (and correspondingly the force) resolution inside a specific region. The multiple mass resolution is implemented in the following way [5]. We first set up a realization of the initial spectrum of perturbations in such a way that initial conditions for a large number $\left(1024^{3}\right)$ of particles can be generated in the simulation box. Initial coordinates and velocities of the particles are then calculated using all waves ranging from the fundamental mode $k=2 \pi / L$ to the Nyquist frequency $k=2 \pi / L \times N^{1 / 3} / 2$, where $L$ is the box size and $N$ is the number of particles in the simulation. Particles outside high-resolution regions are then merged into particles of larger mass and this process can be repeated for merged particles. The larger mass (merged) particle is assigned a velocity and displacement equal to the average velocity and displacement of the smaller-mass particles. Extensive tests of the code and comparisons with other numerical $N$-body codes can be found in [9, 7].

With increasing number of particles and resolution (i.e., number of refinement cells) the memory as well as the computing time requirement of the code increase. In practice, on nodes with $16 \mathrm{~Gb}$ of shared memory and up to 16 CPUs we are limited to simulations with $\leq 256^{3}$ particles (runtime is CPU-bound). The only way to overcome this problem is to use MPI to distribute computations accross nodes. We have developed two different MPI algorithms to handle larger simulations. In the first approach, we select spatially distinct objects of interest and simulate each of these objects on one node with high mass and force resolution whereas in the remaining part of the simulation box lower resolutions will be used. Each node uses then standard ART with OpenMP on shared memory. The inter-node communication is minimal. In the second approach, one can divide the whole simulation box into a number of sub-boxes which will be handled by different nodes. Each node simulates its own sub-box with high mass and force resolution whereas other sub-boxes have lower mass and force resolution. Again one integration step is done by standard ART with OpenMP on each node. After each integration step nodes exchange communications on positions, velocities and masses of particles that cross sub-box boundaries. 


\subsection{Constrained simulations of the local universe}

Here our goal is to perform simulations that match the observed local universe as well as possible. Namely, we are interested in reproducing the observed structures: the Virgo cluster, the Local Supercluster and the Local Group, in the approximately correct locations and embedded within the observed largescale configuration dominated by the Great Attractor and Perseus-Pisces superclusters.

An efficient algorithm for reconstructing the density and velocity fields from sparse and noisy data of redshift and velocity surveys is provided by the Wiener filter formalism and constrained realizations of gaussian random fields [14]. Here we use the MARK III catalog [13]. The sample consists of $\approx 3400$ galaxies and provides radial velocities and inferred distances with fractional errors $\sim 17-21 \%$. A detailed analysis of the large scale structure in the Local Universe reconstructed from the MARK III survey was presented in 16$]$.

Several simulations with increasing force and mass resolution in the region around the Virgo Cluster were performed [6, 10]. The initial conditions for these simulations were set using multiple mass resolution technique. Using $z=0$ output of a low-resolution run, we selected all particles within a sphere of $25 h^{-1} \mathrm{Mpc}$ radius centered on the Virgo cluster. The mass resolution in the Lagrangian region occupied by the selected particles was increased and additional small-scale waves from the initial $\Lambda \mathrm{CDM}$ power spectrum of

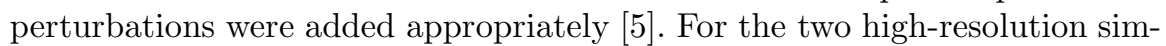
ulations, the particle mass in the Local Supercluster region is 8 and 64 times smaller than in the low-resolution simulation. The highest resolution simulation has a particle mass of $2.5 \times 10^{9} h^{-1} M_{\odot}$ and the maximum formal force resolution was $2.4 h^{-1} \mathrm{kpc}$ in the Local Supercluster region. The results of both high-resolution simulations agree well with each other at all resolved scales.

All major structures (the Local Supercluster, Great Attractor, PerseusPisces supercluster, and Coma cluster) observed within $100 h^{-1} \mathrm{Mpc}$ around the Milky Way exist in the simulations. The positions and morphology of these structures is, of course, fairly well dictated by the constraints imposed on the initial conditions. The Local Supercluster is an elongated structure which extends over $\sim 40 h^{-1} \mathrm{Mpc}$ along the SGX axis. There is a low-density "bridge" (of overdensity just above the average density), which connects the Local Supercluster with the Perseus-Pisces Super-cluster. There is also an even weaker filament connecting the Local Super-cluster with the Great Attractor.

Just as in the real Universe, the Local Group is located in a weak filament extending between the Virgo and Fornax clusters. This filament is a counterpart of the Coma-Sculptor "cloud" in the distribution of nearby galaxies. Figure 11 shows a zoom-in view of the immediate environment of the simulated Local Group. Note that the structures at these scales are only 


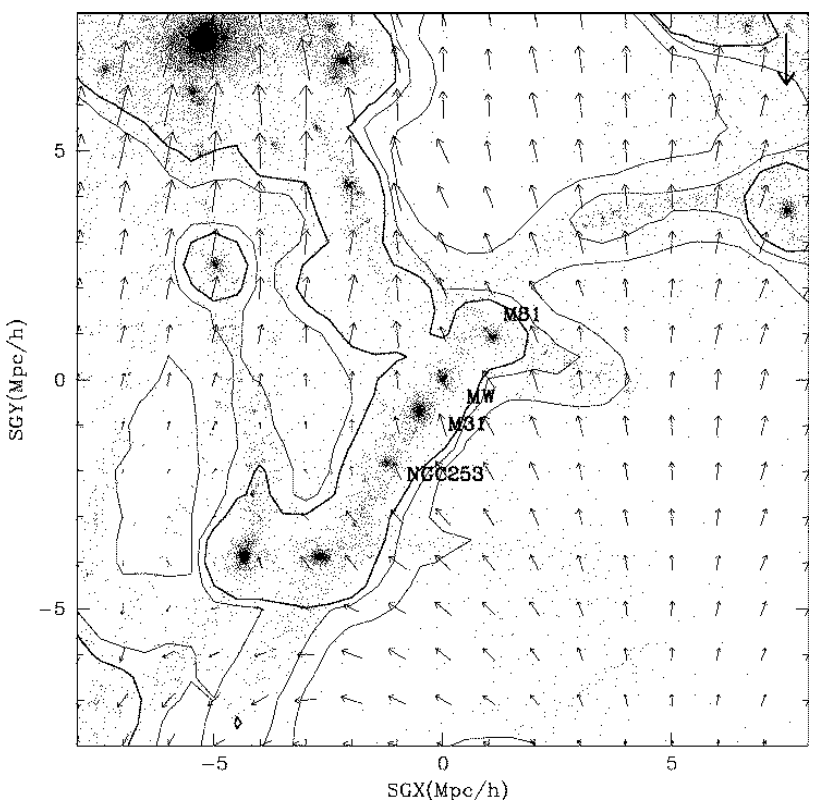

Fig. 1. Density (contours corresponding to overdensities of 1, 2, and 3) and velocity (arrows) fields smoothed with a Gaussian filter of $0.7 h^{-1} \mathrm{Mpc}$ smoothing length around the Local Group. The slice shown has a size and thickness of $15 h^{-1} \mathrm{Mpc}$ and $5 h^{-1} \mathrm{Mpc}$, respectively, and is centered on the supergalactic plane $(S G Z=0)$. The length of the thick arrow in the top right corner corresponds to a velocity of $500 \mathrm{~km} / \mathrm{s}$. The velocities are plotted in the Virgo cluster rest frame.

weakly affected by constraints imposed on the initial conditions. Several possible counterparts to existing objects (e.g., the MW and M31, M51, NGC253) are marked, but their existence is largely fortuitous. As can be seen in this figure, the simulated Local Group is located in a rather weak filament extending to the Virgo cluster. This filament borders an underdense region visible in the right lower corner of Figure 1, which corresponds to the Local Void in the observed distribution of nearby galaxies. We are now carrying out a series of high-resolution simulations of such voids (see below). The velocity field around the Local Group is rather quiet, in good agreement with observations. The peculiar velocity field in the Local Void exhibits a uniform expansion of matter out of this underdense region, while velocities between the Local Group and the Virgo (upper half of Fig. 1) show a coherent flow onto the main body of the Local Supercluster.

\subsection{Galaxy clusters}

Within a low mass resolution simulation $\left(128^{3}\right.$ particles, $m_{\text {part }}=2.0 \times$ $\left.10^{10} h^{-1} M_{\odot}\right)$ we have identified 15 clusters of galaxies with masses above 

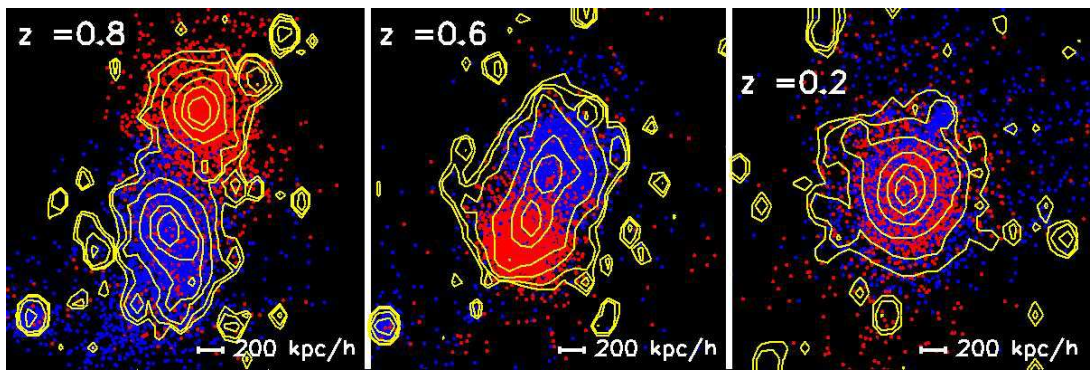

Fig. 2. Merger event between two $6 \times 10^{13} \mathrm{M}_{\odot}$ clusters. Particles belonging to one of the two clusters before merging are plotted with red and blue dots, respectively. Yellow lines correspond to density contours.

$1.0 \times 10^{14} h^{-1} M_{\odot}$. From this set we have selected 8 candidates with different masses and merging histories and added 5 smaller clusters/groups for load balance. We then re-simulated the clusters with higher mass resolution. With particle masses of $3.2 \times 10^{8} h^{-1} M_{\odot}$ a typical cluster and its environment contains more than 1 million particles. The formal force resolution with 9 refinement levels was $1 h^{-1} \mathrm{kpc}$. Halos with masses above $3.0 \times 10^{10} h^{-1} M_{\odot}$ are well resolved. A typical cluster contains more than 150 such halos. The simulations were done using an MPI version of the ART code where each of 8 nodes followed the evolution of one or two clusters. We are now carrying out simulations of these clusters including gasdynamics.

The dynamical evolution of a typical major merger between galaxy clusters can be seen in Fig. 2. The figure shows three snapshots of a cluster taken at $z=0.8, z=0.6$, and $z=0.2$. Although the cluster does not show significant substructure in its density contours during the merger, it is far from a relaxed state. Most of the energy transfer takes place during the first encounter (between $z=0.8$ and $z=0.6$ ), but the merger remnant does not reach virial equilibrium until it loses all the information about the initial conditions (i.e. the particles are well mixed). For this event, virialisation occurs at $z \sim 0.2(\sim 4$ Gyr after the first encounter). During the first stages of cluster formation, the characteristic time between major mergers can be shorter than the relaxation time. High-redshift clusters may thus be in general far from the virial equilibrium inside their formal virial radius.

\subsection{Voids}

Cosmological simulations predict many more small DM halos than the observed number of satellites around the Milky Way and Andromeda galaxies. Do we have the same problem for dwarf galaxies in voids? One naively expects a large number because the Press-Schechter mass function steeply rises with declining mass. In contrast, it seems that observations are failing to find a substantial number of dwarf galaxies inside voids (e.g. [11, 3]). However, the 


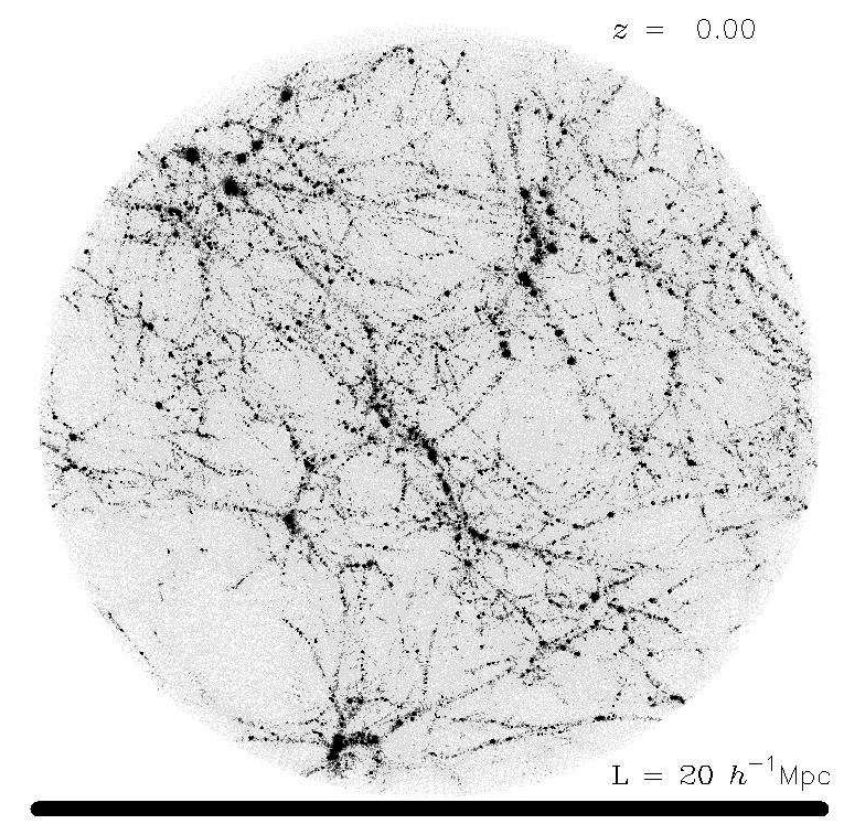

Fig. 3. The inner region of a spherical void at $z=0$.

situation is complicated because it is very difficult to detect dwarf galaxies, many of them are expected to have low surface brightness.

In order to study the formation of large voids, the simulation box should be sufficiently large; we use a cube of size $80 h^{-1} \mathrm{Mpc}$. On the other hand, we are interested in the formation of small structure elements inside voids, for which we need highest possible mass resolution. Therefore, we use particles with large masses to follow the evolution of large scale structures and particles with small masses to follow the evolution of structure within one spherical void.

Within a low mass resolution simulation $\left(128^{3}\right.$ particles, $m_{\text {part }}=2.0 \times$ $\left.10^{10} h^{-1} M_{\odot}\right)$ we have identified 8387 galactic halos with masses $>2.0 \times$ $10^{11} h^{-1} M_{\odot}$. This corresponds to a mean distance of about $4 h^{-1} \mathrm{Mpc}$ between halos. We then searched voids in the distribution of these galactic halos by constructing the minimal spanning tree using halo positions. The minimal spanning tree was then used to search for the point in the simulation box which has the largest distance $r_{1}$ to the set of halos. We identified this point as the center of spherical void with the radius of $r_{1}$. Excluding that void we were searching again for the point with the largest distance to the set and thus found the second largest void and so on. The algorithm is similar to that used by [1].

With the algorithm described above we find spherical voids in the halo distribution which do not contain any halo with a mass larger than $2.0 \times$ $10^{11} h^{-1} M_{\odot}$; such halos by definition lie on the border of the void. After finding voids in the low-resolution simulation, we re-run the simulation with 
much higher mass resolution inside the voids $\left(m_{\text {part }}=4.0 \times 10^{7} h^{-1} M_{\odot}\right)$, which allows identification of objects with masses larger than $10^{9} h^{-1} M_{\odot}$. By construction, the voids do not have halos with masses larger than $M_{\mathrm{b}}=$ $2.0 \times 10^{11} h^{-1} M_{\odot}$. Five voids have been identified and resimulated, their radii are $r_{\text {void }}=11.6,10.8,9.4,9.1,9.1 \mathrm{~h}^{-1} \mathrm{Mpc}$. Inside the voids the matter density is typically a factor of 10 smaller than the mean density, but void properties exhibit large differences. Some voids are very isolated: the density within a sphere with radius $30 h^{-1} \mathrm{Mpc}$ centered on one of the void centers reaches only $2 / 3$ of the mean density. On the other hand, the most prominent structure of the simulation, a galaxy cluster of $2 \times 10^{15} h^{-1} \mathrm{Mpc}$, is bordering one of the other voids. The density outside of this void rapidly increases. The mass function in voids is about an order of magnitude lower and its shape is different than that of the field galaxies [2].

In Fig. 3 we show the inner $20 h^{-1} \mathrm{Mpc}$ of a spherical void of radius 21.6 $h^{-1} \mathrm{Mpc}$. In this void we found more than 50 halos with circular velocity $v_{c}>50 \mathrm{~km} / \mathrm{s}$ and more than 600 halos with $v_{c}>20 \mathrm{~km} / \mathrm{s}$. There is a certain spatial mass segregation among halos. Typically, more massive halos tend to be situated in the outer part of the void. The largest halos in the plot are actually dwarf-size halos with circular velocities of $\sim 50 \mathrm{~km} / \mathrm{s}$. The void is far from being empty and boring: it has a complex structure with numerous long filaments and small sub-voids. Visually it resembles the large-scale structure of the Universe, but everything in this plot is hundreds and thousands times less massive than in "normal" configurations of interconnected superclusters and filaments.

\subsection{Hydro simulations}

We have started to repeat [10] many of the simulations described above including eulerian shock-capturing gasdynamics and physical processes such as radiative cooling and stellar feedback. Inclusion of gasdynamics, although significantly increasing computational and memory demands of simulations, will allow us to address a much wider range of questions and more robust comparison with observations. For example, simulations of the Local Supercluster regions with cooling and starformation will allow us to study distribution and properties of galaxies in the simulations as a function of their luminosity and color. High-resolution simulations of the local voids will allow studies of spatial distribution of Ly $\alpha$ absorbers and their connection to galaxies. Simulations of nearby galaxy clusters (e.g., Virgo, Fornax, Coma) formed in realistics large-scale environments will allow for detailed object-to-object comparison of properties and should give us good insights into physical processes operating within intracluster medium.

As an example, we present here simulations of the Virgo cluster in the context of constrained simulations described in $\S 2.1$. The lagrangian region within five virial radii (at $z=0$ ) around the cluster was resimulated with 

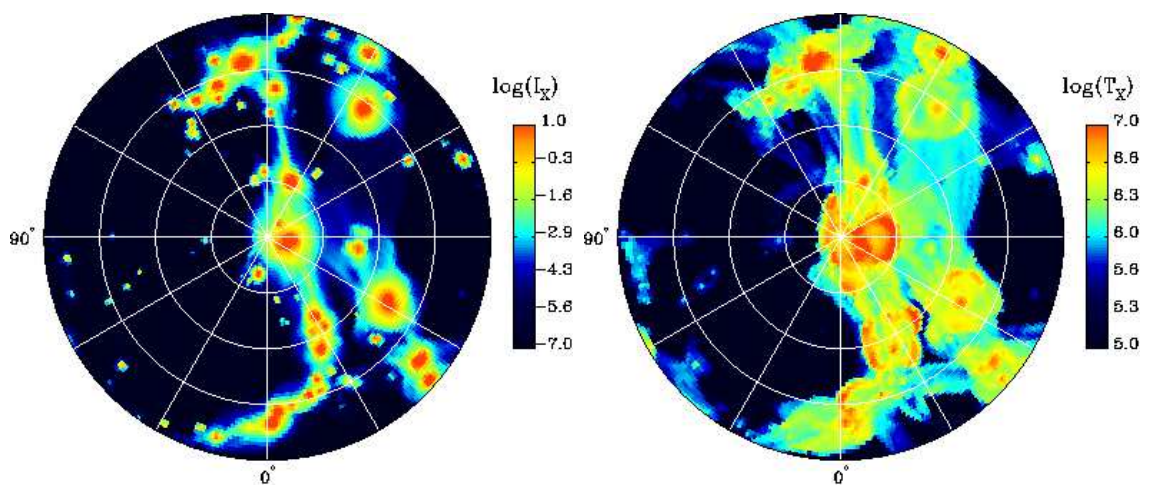

Fig. 4. The projected X-ray intensity and emission-weighted temperature maps of the Local supercluster in galactic coordinates. The Virgo cluster is the brightest object near the center (which corresponds to the North Galactic Pole).

mass resolution 8 times higher (particle mass of $3.1 \times 10^{8} h^{-1} M_{\odot}$ ) than that of the Local Supercluster simulation and spatial resolution of $\approx 1 h^{-1} \mathrm{kpc}$ in the central regions of the cluster. Figure 1 shows the sky projection of the $\mathrm{X}$-ray intensity and emission-weighted temperature of gas in the simulations in galactic coordinates (the center of the polar projection corresponds to the North Galactic Pole, NGP). The Virgo cluster is the brightest object located near the NGP, very close to the actual location of the real Virgo cluster. Figure a shows radial profiles of dark matter and gas density, as well as profiles of gas temperature and entropy for two simulations: one that included only adiabatic gasdynamics, and the other in which gas was preheated with the energy of $1.5 \mathrm{keV}$ per gas particle at $z=3$. The preheating very roughly models the possible effect of galactic winds at high-redshifts. The figure shows that preheating increases entropy of the gas thereby lowering its density in the cluster core and increasing the overall gas temperature. It lowers the X-ray luminosity of the cluster by a factor of 8 , which brings it in good agreement with the observed luminosity-temperature relation. Note, however, that it does not change the shape of the temperature profile. The proximity of the Virgo cluster makes it one of the best spatially resolved clusters, with the temperature mesurements well outside the virial radius. Figure 6 compares the observed temperature measurements around the center of the Virgo cluster 12 as a function of projected radius to the corresponding measurements in simulations (adiabatic and preheating). We constructed projected map of emission-weighted temperature of the simulated Virgo cluster using $4^{\prime} \times 4^{\prime}$ pixels, each pixel represented by a point in the figure. The figure shows that neither simulation can match the much shallower temperature distribution in the central regions of the Virgo cluster. This indicates that preheating alone cannot be the whole story and other processes, such as cooling and/or central 

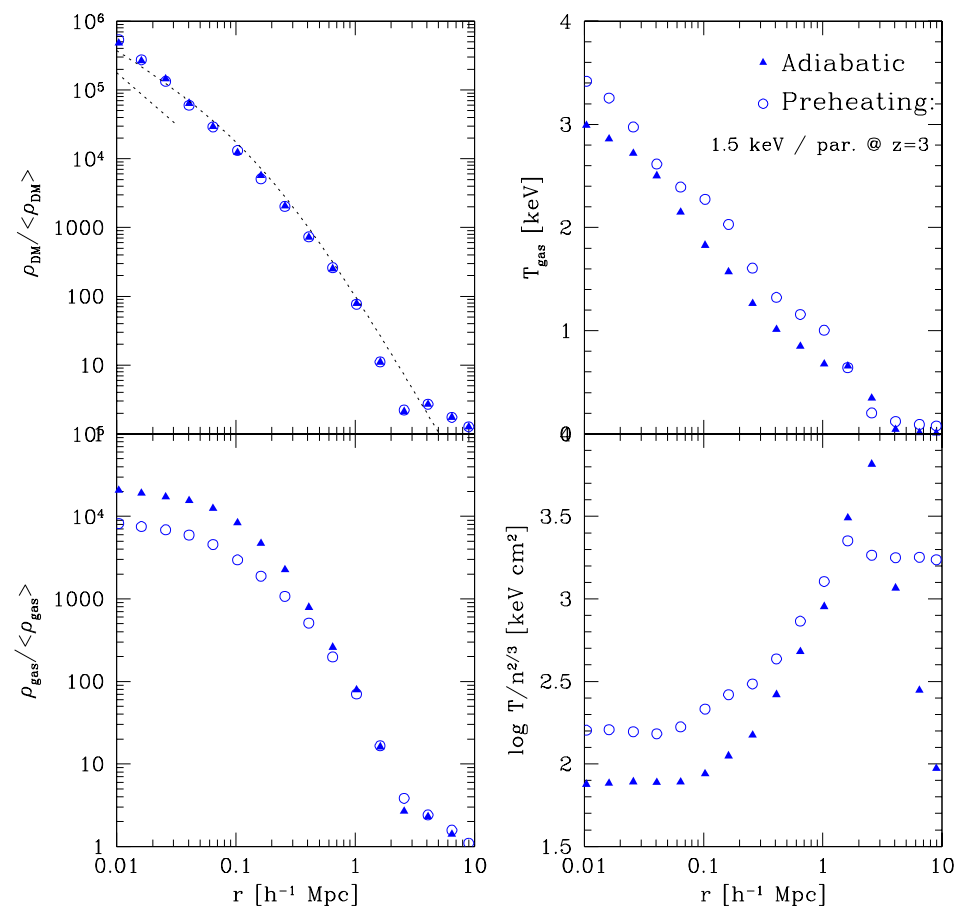

Fig. 5. Radial profiles of dark matter and gas density, gas temperature, and gas entropy of the simulated Virgo cluster. The dotted line in the DM density panel shows the NFW profile with the concentration of 7 .

heating by AGNs can be important. We will be exploring the effects of these processes in future simulations.

\section{Summary}

The adaptive refinement tree code is a useful tool to study cosmological structure formation on different scales and with different resolutions. It runs well on a variety of platforms with shared and distributed memory. The simulations described here have been performed on the Hitachi of LRZ Munich, the small development Hitachi at the AIP, the IBM SP of the Potsdam Institute for Climate Impact Research, and the IBM SP of NERSC Berkeley.

\section{References}

1. Einasto, J., Einasto, M., \& Gramann, M., 1989, 238, 155 


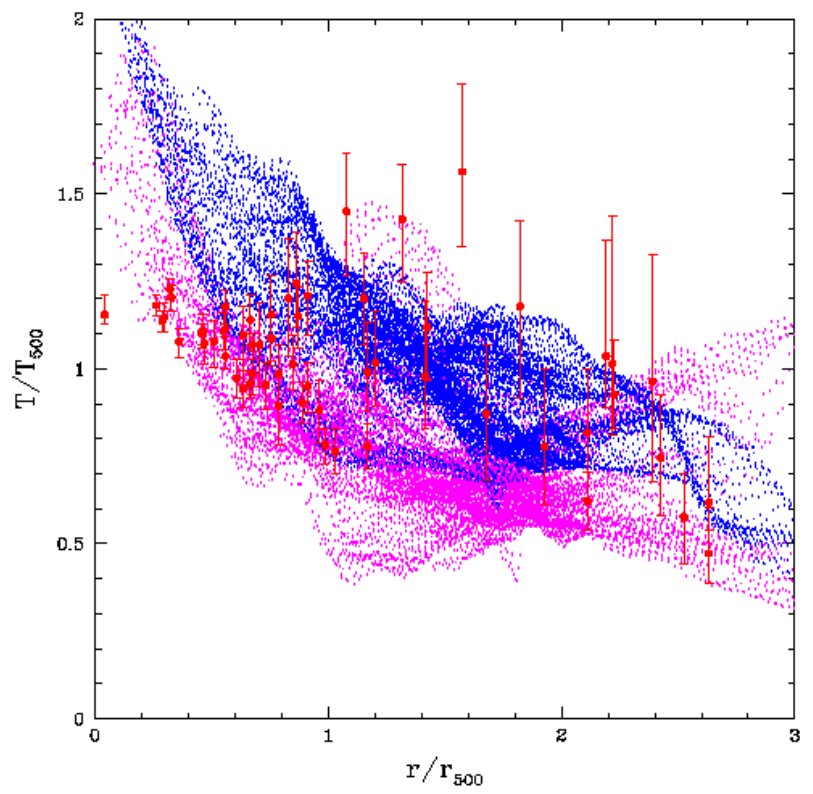

Fig. 6. Projected temperature profiles of the observed (points with errorbars) and simulated (purple and blue points) Virgo cluster. Purple points correspond to the adiabatic simulation while blue points show temperature in the simulation with preheating. The radii are normalized to the radius corresponding to the overdensity of 500 with respect to the critical density.

2. Gottlöber, S., Łokas, E.,Klypin, A., astro-ph/0207487

3. Grogin, N.A., Geller, M.J., 2000, Astrophys. J. 119, 32

4. Khokhlov, A.M. 1998, J. Comp. Phys. 143, 519

5. Klypin, A., Kravtsov, A. V., Bullock, J. S., \& Primack, J. R. 2001, Astrophys. J. 554, 903

6. Klypin, A., Hoffman, Y., Kravtsov, A. V., \& Gottlöber, S. 2001, astroph/0107104, submitted to Astrophys. J.

7. Knebe, A., Kravtsov, A. V., Gottlöber, S., \& Klypin, A. A. 2000, MNRAS 317, 630

8. Kravtsov, A. V., Klypin, A. A., \& Khokhlov, A. M. 1997, Astrophys. J. Suppl. 111,73

9. Kravtsov, A. V. 1999, PhD thesis, New Mexico State University

10. Kravtsov, A. V., Klypin, A., Hoffman, Y., 2002, Astrophys. J. 571, 563

11. Popescu, C.C., Hopp, U., \& Elsässer, H. 1997, A\&A 325, 881

12. Shibata, R. et al. 2001, Astrophys. J. 549, 228

13. Willick, J. A., Courteau, S., Faber, S. M., Burstein, D., Dekel, A., \& Strauss, M. A. 1997, Astrophys. J. Suppl., 109, 333

14. Zaroubi, S., Hoffman, Y., Fisher, K. B., \& Lahav, O. 1995, Astrophys. J., 449, 446

16. Zaroubi, S., Hoffman, Y., \& Dekel, A. 1999, Astrophys. J. 520, 413 